\title{
Emerging Multiple Parasite Reinfection as Neglected Tropical Disease: A Case Series
}

\author{
Fatima Safira Alatas ${ }^{1}$ Aldisa Ayu Pratiwi ${ }^{1}$ Agnes Kurniawan ${ }^{2}$ Antonius Hocky Pudjiadi ${ }^{1,3}$ (1) \\ ${ }^{1}$ Department of Child Health, Cipto Mangunkusumo Hospital, Faculty \\ of Medicine, Universitas Indonesia, Jakarta, Indonesia \\ 2 Department of Parasitology, Faculty of Medicine, Universitas \\ Indonesia, Jakarta, Indonesia \\ Address for correspondence Fatima Safira Alatas, MD, PhD, \\ Department of Child Health, Cipto Mangunkusumo Hospital, Faculty \\ of Medicine, Universitas Indonesia, Jl. Diponegoro No. 71, Jakarta, \\ Indonesia (e-mail: safiraalatas@gmail.com).
}

${ }^{3}$ Scientific Affair of Indonesian Pediatric Society Head Office, Jakarta, Indonesia

J Child Sci 2022;12:e9-e12.

\begin{abstract}
Keywords

- trichuriasis

- multiple helminth infections

- gastrointestinal bleeding

- growth faltering

Soil-transmitted helminth infection is endemic in Indonesia, with cases of reinfection despite individualized therapy and a mass treatment program. Three cases of multiple helminth infections were described that might be associated with chronic bloody diarrhea and stunted growth. These case series alert pediatricians that untreated helminthic infections are part of the differential diagnosis in children with gastrointestinal bleeding. Environmental surveillance should be performed in high-risk areas to prevent reinfection and growth alteration. In endemic areas, bloody diarrhea warns for an early routine fecal analysis. Correct diagnosis, prompt treatment, and source control are crucial to prevent long-term complications.
\end{abstract}

\section{Introduction}

Soil-transmitted helminth (STH) reinfections occur commonly after treatment and are still a major neglected tropical disease in developing countries. STH consists of Ascaris lumbricoides, Trichuris trichiura, and hookworm (Necator americanus and Ancylostoma duodenale). T. trichiura, or whipworm, is one of the four most common types of nematode worms affecting 463.7 million humans globally.,2 Prevalence of worm infections in rural areas in Indonesia reaches more than $25 \%$, with $24.6 \%$ observed among primary school children in Java, Indonesia. ${ }^{3}$ Most STHs have common symptoms including growth stunting and cognitive restriction. In addition, Trichuriasis can manifest as severe disease with chronic diarrhea or dysentery syndrome, abdominal pain, nausea, vomiting, severe iron deficiency anemia, and rectal prolapse. ${ }^{4}$ Reinfection of STH can occur despite individualized treatment and a mass treatment program as preventive therapy. It is closely associated with poverty, overcrowding, poor sanitation, and low health education

received

April 5, 2021

accepted after revision

December 20, 2020 level. This case series highlights STH reinfection occurring in lower socio-economic demographics in Indonesia.

\section{Case Presentation}

\section{Case 1}

Patient A, a 17-year-old male, was referred from West Java to Cipto Mangunkusumo Hospital for the evaluation of persistent bloody diarrhea for 10 years before admission. The suspected cause was rectal polyposis. The patient complained of foul-smell stool and white worms coming out of the anus and rectal lump during defecation. He had received serial blood transfusion for chronic recurrent anemia. Physical examination showed normal vital signs with microcephaly (head circumference $<2$ standard deviation and severe stunting (weight and height were $<\mathrm{P} 3$ National Center for Health Statistics curve Centers for Disease Control and Prevention 2000); however, nutritional status was good (weight/height was 91\%). Rectal examination showed no polyp/mass.

\section{(c) 2022. The Author(s).}

This is an open access article published by Thieme under the terms of the Creative Commons Attribution License, permitting unrestricted use, distribution, and reproduction so long as the original work is properly cited. (https://creativecommons.org/licenses/by/4.0/)

Georg Thieme Verlag KG, Rüdigerstraße 14, 70469 Stuttgart, Germany 


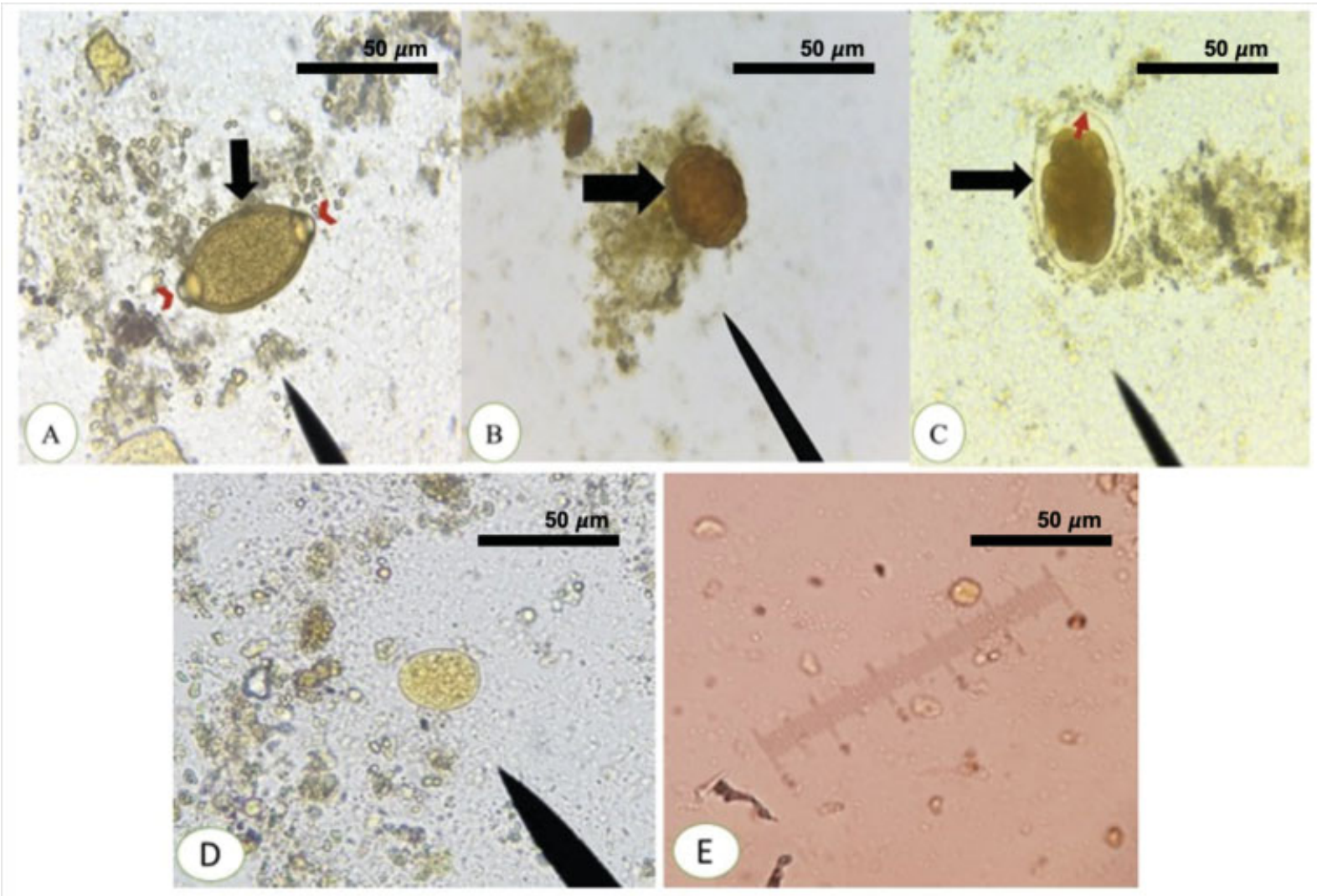

Fig. 1 Light microscopy of helminth eggs observed in parasitological stool analysis (A) Trichuris trichiura egg, showing barrel shape/lemon shape/football shape; the shell is thick and smooth, yellowish-brown in color, translucent hyaline polar plug at each end [red head arrows],(B) Ascaris lumbricoides egg, showing rough and bumpy outer surface with brownish color, and (C) hookworm egg, showing oval-round shaped, thin with smooth and colorless (transparent) eggshell, clear space between the eggshell and the ovum [thin red arrows in inner surface], and (D) Entamoeba coli cyst and (E) vacuolar form of Blastocystis spp.

Laboratory tests showed normal hemoglobin, platelet count, leucocyte count, and blood clotting function. Parasitological stool examination using light microscopy showed eggs of T. trichiura, A. lumbricoides, and hookworm and cysts of Entamoeba hystolitica/dispar and Entamoeba coli ( - Fig. 1). Colonoscopy examination showed hundreds of worms covering the mucosa of the colon, and no polyp was identified ( - Fig. 2A, B). The patient was diagnosed with severe trichuriasis and multiple intestinal parasites infections. Mebendazole was administered at $100 \mathrm{mg}$ twice daily for
5 days, and metronidazole at $500 \mathrm{mg}$ three times daily for 10 days. Clinical symptoms improved after 5 days treatment, supported by no parasites found on re-evaluation.

\section{Case 2}

Patient N, a 5-year-old male, was referred to Cipto Mangunkusumo Hospital due to suspicion of rectal polyposis. He suffered from bloody diarrhea for 2 years before admission and also had a serial blood transfusion. He also had white worms coming out from the anus and rectal lump while
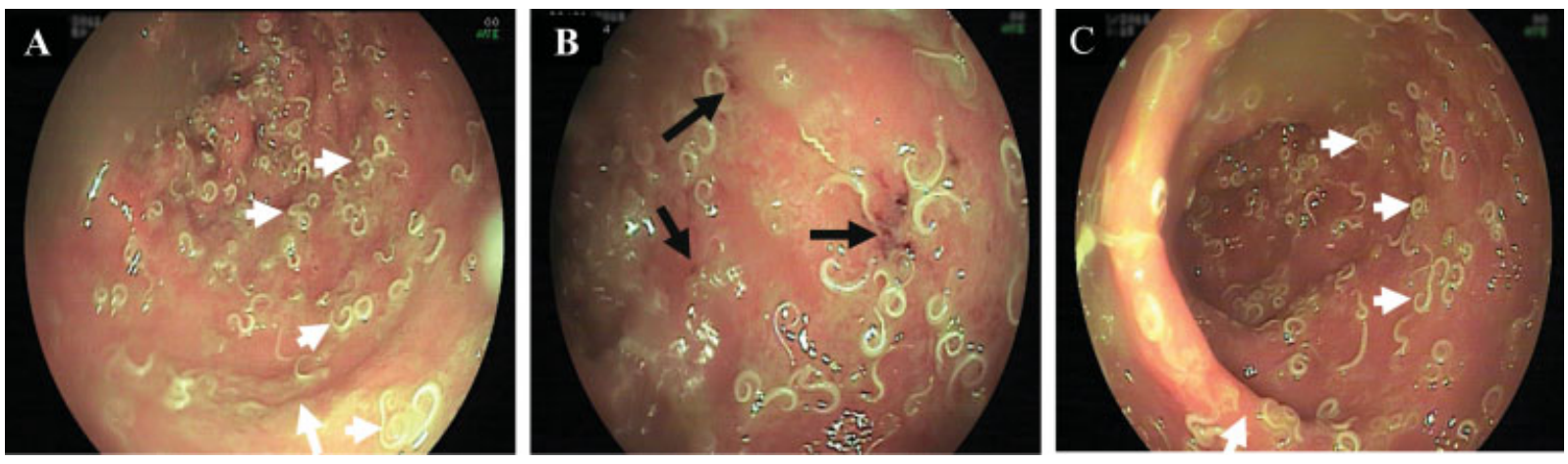

Fig. 2 Colonoscopy showing whitish worms (Trichuris trichiura) in the colon. (A and B) Images of case 1 showing whitish worms (white arrow) in the mucosa of colon and black arrows showing mucosal erosion with bleeding spot. (C) Images of case 2, showing worm, Trichuris trichiura (white arrow), with coiled posterior region. 
defecation. Physical examination showed normal vital signs, mild malnourishment, and no polyp/mass observed on rectal examination. Hematology examination showed normal hemoglobin, platelet count, and blood clotting function, but leucocyte count was high. Parasitological stool examination using light microscopy showed eggs of T. trichuria, A. lumbricoides, and hookworm and cysts of E. hystolitica/dispar and E. coli. Colonoscopy examination also showed hundreds of worms in the colon and no polyp was identified. (-Fig. 2C).

The patient also was diagnosed with severe trichuriasis and multiple intestinal parasites infections. We administered mebendazole at $100 \mathrm{mg}$ two times daily and metronidazole at $240 \mathrm{mg}$ three times daily for 5 days. Clinical symptoms also improved after five days of treatment, and no parasites were found on re-evaluation.

\section{Case 3}

Patient AL, a 13-year old male, was the sibling of patient A and N. Patient had no gastrointestinal complained; however, we performed parasites stool examination for screening purpose. Interestingly, evaluation result showed positive eggs of A. lumbricoides, T. trichiura, hookworm, and vacuolar form of Blastocystis spp. (-Fig. 1E). The patient was treated with the same medications as cases 1 and 2 . The patient reported improved clinical symptoms, and no parasites were found on re-evaluation examination.

We identified a slight difference in all three cases. Case 1 and case 2 showed major long-term consequences of untreated chronic multiple helminths infections, whereas case 3 showed mild STH infection without any symptom present.

\section{Six Months of Follow-up after Treatment}

We performed a new parasitological stool examination 6 months after treatment on each patient. Case 1 had positive eggs of T. trichiura, A. lumbricoides, and B. hominis, while case 2 and 3 had both eggs and adult worms of T. trichiura and hookworm. Evaluation of additional family members (parents and one other sibling) showed positive multiple parasite infections (eggs of T. trichiura, A. lumbricoides, and hookworm and cysts of Giardia lamblia and Blastocystis hominis). Laboratory tests were not performed. All family members started their treatment with mebendazole with additional metronidazole as conjunctive treatment.

\section{Discussions}

Without adequate treatment, trichuriasis could cause severe infection resulting in chronic bloody diarrhea, rectal prolapse, malnutrition, short stature/stunting, and severe anemia due to chronic bowel mucosa infection. ${ }^{5}$ As shown in case 1 , chronic malnutrition occurred due to 10 years of inadequate parasitic treatment, resulting in stunting and microcephaly. ${ }^{6}$ Anal lumps due to rectal prolapse are often caused by increased intra-abdominal pressure during diarrhea and irritation of bowel mucosa caused by severe trichuriasis infection. Trichuriasis infection could cause loss of red blood cells directly from the gut, followed by iron deficiency anemia and growth faltering. Thus, increased tumor necrosis factor- $\alpha$ as an inflammatory mediator is thought to inhibit patient's appetite and interfere with metabolic processes. ${ }^{7}$

The patients' families came from low economic status, had low education, lived in an environment with poor sanitation (e.g., lack of hand washing before meal and walking barefoot), limited water supply, and grounded house, with defecation habits in inappropriate places (e.g., land or river) in consequence of unavailability of the private latrine. These lifestyles further caused multiple parasitic reinfections in all family members although effective parasitic treatment had been achieved. The availability of latrine, periodic health evaluation and education, and better hygiene promotions by health care providers will reduce infections. ${ }^{3}$

Treatment of trichuriasis requires the combination of at least two anthelmintic regiments, as monotherapy was deemed unsatisfactory. ${ }^{8}$ Albendazole monotherapy resulted in the lower cure rate compared with mebendazole (2.6 vs. $11.8 \%$ ) for trichuriasis. ${ }^{9}$ However, albendazole is a drug of choice for hookworms and A. lumbricoides infection. In triple infections cases, oxantel pamoate-albendazole regimen was reported to be superior to mebendazole, with a cure rate of 31.2 versus $11.8 \%$ and egg reduction of 96 versus $75 \%{ }^{9}$ Oxantel pamoate has low efficacy in eradicating hookworm and $A$. lumbricoides. Single-dose mebendazole had a cure rate of $92.6 \%$ for A. lumbricoides, $27.5 \%$ for T. trichiura, and $25.5 \%$ for hookworms, and high egg-reduction rate for all three cases. $^{10}$

In Cuba, mebendazole $500 \mathrm{mg}$ twice a year was used for mass drug administration in the eradication of trichuriasis. ${ }^{11}$ This is in accordance with our serial cases showing 5 days of mebendazole monotherapy treatment effective in curing multiple helminths infections, as proved by parasite stool re-evaluation showing negative results in all cases. ${ }^{10}$ Moreover, albendazole availability in Indonesia is limited to primary health centers. Furthermore, combination regimens were not used in the cases above to prevent anthelmintic resistance. Metronidazole is only used in patients with E. histolytica co-infection who are also suspected of having bacterial overgrowth.

Reinfection can occur rapidly after treatment with standard regiment, particularly for A. lumbricoides and T. trichiura. ${ }^{12}$ Reinfection was higher in stunted children, with inadequate STH treatment, poor sanitation/hygiene, and scarce clean water supplies. ${ }^{13,14}$ Following the administration of albendazole-oxantel pamoate treatment, it was reported that post-treatment the reinfection rate of $A$. lumbricoides reached $34.6 \%$ and $T$. trichiura $37.2 \%$ in 18 weeks. ${ }^{12}$ Other strategies to control STH infections include regular deworming of school-aged children, which is considered to be more cost-effective in settings where resources are limited. In addition to reducing reinfection rate, long-term solutions needed include improvement in water quality, sanitation, hygiene, poverty reduction, health education, and attention to chronic malnutrition. ${ }^{13-15}$ The parents were educated for the prevention of reinfection before returning to their hometown. 


\section{Conclusions}

T. trichiura may result in severe infection such as chronic bloody diarrhea, recurrent abdominal pain, severe anemia, loss of appetite, weight loss, rectal prolapse, malnutrition, and severe stunting. Bloody diarrhea should be evaluated early by routine fecal analysis and colonoscopy if possible and treated promptly to prevent long-term complications. Recurrent infections of helminths and parasites, especially in low-educated and poor hygiene areas, should be prevented by continuous health evaluation and education by local health care providers. Health education should be incorporated in school curricula, which will inculcate awareness among the younger generation and eventually disseminating to families and larger communities. In addition, cooperation with the local government in regulating environmental household programs is also essential. Periodic helminths evaluation should be done by local health care providers in endemic areas for early diagnosis, and anthelmintic regiment should be given if needed. Treating worm infection without proper source controlling was impractical and not economical.

\section{Consent for Publication}

Written informed consent was obtained from the patient for the publication of this case report and accompanying images.

\section{Authors' Contributions}

F.S.A. coordinated the writing of the case series and was the attending senior consultant who examined the patient and performed a colonoscopy examination. A.A.P. helped in the drafting of the manuscript and follow-up of the patient, A.K. helped with parasitology examination of the patients and writing of discussion, and A.H.P. helped to draft the manuscript and supervise the project. All authors have read and approved the final version of the manuscript.

\section{Funding}

None.

\section{Conflict of Interest \\ None declared.}

\section{Acknowledgments}

We would like to acknowledge Anandya Anton Atmojo Hadi, M.D. for collecting the patient's data and managing patients and family in outpatient's clinic. Finally, we acknowledge the patients and their families for their willingness to share their clinical data for this case series. We would also like to thank the Department of Parasitology, Faculty of Medicine Universitas Indonesia for the evaluation of fecal samples for all family members, and the Indonesian Pediatric Society in supporting community health care in preventing stunting in Indonesian children.

\section{References}

1 Bethony J, Brooker S, Albonico M, et al. Soil-transmitted helminth infections: ascariasis, trichuriasis, and hookworm. Lancet 2006; 367(9521):1521-1532

2 Weatherhead JE, Hotez PJ, Mejia R. The global state of helminth control and elimination in children. Pediatr Clin North Am 2017; 64(04):867-877

3 Park MJ, Laksono B, Clements A, Sadler R, Stewart D. Worm-free children: an integrated approach to reduction of soil-transmitted helminth infections in Central Java. Rev Environ Health 2016;31 (01):111-113

4 Diniz-Santos DR, Jambeiro J, Mascarenhas RR, Silva LR. Massive Trichuris trichiura infection as a cause of chronic bloody diarrhea in a child. J Trop Pediatr 2006;52(01):66-68

5 Zanwar VG, Pawar SV, Jain SS, Rathi SP, Contractor QQ Rathi PM. An unusual cause of overt gastrointestinal bleeding in a malnourished child. Trop Doct 2016;46(02):100-102

6 Tiwari K, Goyal S, Malvia S, Sanadhya A, Suman RL, Jain R. Impact on malnutrition on head size and development quotient. Int J Res Med Sci 2017;24:3003-3006

7 Zeehaida M, Zueter A, Zairi NZ, Zunulhisham S. Trichuris dysentery syndrome: do we learn enough from case studies? Trop Biomed 2015;32(03):545-550

8 Keiser J, Utzinger J. Efficacy of current drugs against soil-transmitted helminth infections: systematic review and meta-analysis. JAMA 2008;299(16):1937-1948

9 Speich B, Ame SM, Ali SM, et al. Oxantel pamoate-albendazole for Trichuris trichiura infection. N Engl J Med 2014;370(07):610-620

10 Mrus J, Baeten B, Engelen M, Silber SA. Efficacy of single-dose $500 \mathrm{mg}$ mebendazole in soil-transmitted helminth infections: a review. J Helminthol 2018;92(03):269-278

11 van der Werff SD, Vereecken K, van der Laan K, et al. Impact of periodic selective mebendazole treatment on soil-transmitted helminth infections in Cuban schoolchildren. Trop Med Int Health 2014;19(06):706-718

12 Speich B, Moser W, Ali SM, et al. Efficacy and reinfection with soiltransmitted helminths 18 -weeks post-treatment with albendazole-ivermectin, albendazole-mebendazole, albendazole-oxantel pamoate and mebendazole. Parasit Vectors 2016;9:123

13 Halpenny CM, Paller C, Koski KG, Valdés VE, Scott ME. Regional, household and individual factors that influence soil transmitted helminth reinfection dynamics in preschool children from rural indigenous Panamá. PLoS Negl Trop Dis 2013;7(02):e2070

14 Strunz EC, Addiss DG, Stocks ME, Ogden S, Utzinger J, Freeman MC. Water, sanitation, hygiene, and soil-transmitted helminth infection: a systematic review and meta-analysis. PLoS Med 2014;11 (03): 1001620

15 Jia TW, Melville S, Utzinger J, King CH, Zhou XN. Soil-transmitted helminth reinfection after drug treatment: a systematic review and meta-analysis. PLoS Negl Trop Dis 2012;6(05):e1621 